\title{
On frame and orientation localization for relative sensing networks
}

Giulia Piovan

Iman Shames
Barış Fidan
Francesco Bullo
Brian D. O. Anderson

\begin{abstract}
We develop a novel localization theory for planar networks of nodes that measure each other's relative position, i.e., we assume that nodes do not have the ability to perform measurements expressed in a common reference frame. We begin with some basic definitions of frame localizability and orientation localizability. Based on some key kinematic relationships, we characterize orientation localizability for networks with angle-of-arrival sensing. We then address the orientation localization problem in the presence of noisy measurements. Our first algorithm computes a least-square estimate of the unknown node orientations in a ring network given angle-ofarrival sensing. For arbitrary connected graphs, our second algorithm exploits kinematic relationships among the orientation of node in loops in order to reduce the effect of noise. We establish the convergence of the algorithm, and through some simulations we show that the algorithm reduces the meansquare error due to the noisy measurements.
\end{abstract}

\section{INTRODUCTION}

One of the key problems in sensor networks is localization, i.e., determining the location of each sensor in the network. Sensor networks are used in a large number of applications which cover a wide range of fields, such as, surveillance, targeting systems, controls, communications, monitoring areas, intrusion detection, vehicle tracking and mapping.

We address the problem in a distributed manner, by assuming that any node in the network has its own reference frame, and does not have any knowledge about its physical position in the environment or the position of all the other nodes. Each node, through a sensor, can detect the presence and the relative position of any node inside a given sensor footprint. The measures are affected by noise, so we extend our analysis to the noisy case. We call frame localization the problem of computing the relative location and orientation of each node of the network. We aim to solve the problem through a distributed algorithm, which computes the estimate of the angle associated to every edge of the graph by distributing the error of every cycle on its edges.

Network localization has been the center of extensive research work, and the various approaches are due to different assumptions on the deployment of the nodes and the way sensors work. In some cases, there is the use of some special nodes, whose position is known, called beacons or anchors. Moreover, to obtain a more accurate estimation, some researchers, e.g., [1], investigate the possibility of choosing the position of the beacons. Particular interest arises from the works of Roumeliotis and coworkers, [2] and [3], in

G. Piovan and F. Bullo are with the Department of Mechanical Engineering, University of California at Santa Barbara, Santa Barbara, CA 93106, USA, \{giulia, bullo\}@engineering.ucsb.edu, I. Shames, B. Fidan, and B. D. O. Anderson are with Australian National University and National ICT Australia, Canberra, ACT 0200, Australia, \{Iman.Shames, Baris.Fidan\}eanu.edu.au and Brian. Andersoneanu . edu.au. which the problem of determining the relative position for pair of robots moving in $2 \mathrm{D}$ or $3 \mathrm{D}$ is studied using only distance measurements between the robots. The literature review includes also the beautiful treatment in [4], where a theory of localization emerges.

This paper contains several contributions and proceeds as follows. First, we review and identify a few useful kinematic relationships and fundamental elements of graph theory. Second, we present a novel formulation of the frame localizability and frame computational localization problem for networks with relative sensing. Third, we define a characterization of frame localizability for planar networks, focusing on consistency for the orientation localization problem. Fourth, we compute a least-square estimate of the unknown node orientations in a ring network. Fifth, we consider arbitrary connected graphs and provide a distributed algorithms for planar orientation localization which exploits kinematic relationships among the orientation of nodes in loops in order to reduce the effect of noise. We also add some considerations on the convergence rate of the algorithm. Finally, we provide some simulations in order to validate our algorithm results.

The paper is organized as follows. In Section II, we review some kinematic conventions and elements of graph theory that are used throughout the paper. Section III is dedicated to the description of the network model and the problem with some preliminary relationships on relative positions. Section IV studies the orientation localizability of the network considering the measurement noise, including a formula to compute the optimal least-squares angle estimate in a ring, and offers a convergent algorithm for the frame localization problem in a general network. In Section V some simulation results are shown. Section VI gives a short review of the paper with conclusions and aims for future work. All proofs can be found in the technical report [5]

\section{Preliminaries}

\section{A. Elements of kinematics}

We let $\mathbb{R}$ and $\mathbb{C}$ denote real and complex numbers, respectively. We let $\|\boldsymbol{v}\|$ denote the Euclidean norm of the vector $\boldsymbol{v} \in \mathbb{R}^{d}$. We define the versor operator vers : $\mathbb{R}^{d} \rightarrow \mathbb{R}^{d}$ by $\operatorname{vers}(\mathbf{0})=\mathbf{0}$ and $\operatorname{vers}(\boldsymbol{v})=\boldsymbol{v} /\|\boldsymbol{v}\|$ for $\boldsymbol{v} \neq \mathbf{0}$. Given a scalar $\theta$, we let $\operatorname{proj}(\theta)$ take value in $[-\pi, \pi[$, where the map proj: $\mathbb{R} \rightarrow[-\pi, \pi[$ is defined by

$$
\operatorname{proj}(\theta)=(\theta+\pi) \bmod 2 \pi-\pi .
$$

We let $\angle z$ denote the phase of $z \in \mathbb{C}$.

We will be interested in measurements expressed in different reference frames. Accordingly, it is useful to review some basic kinematic conventions. We let $\Sigma_{1}=\left\{p_{1}, \boldsymbol{x}_{1}, \boldsymbol{y}_{1}, \boldsymbol{z}_{1}\right\}$ 
be a fixed reference frame in $\mathbb{R}^{3}$. A point $q$ and a vector $\boldsymbol{v}$ expressed with respect to frame $\Sigma_{1}$ are denoted by $q^{1}$ and $\boldsymbol{v}^{1}$, respectively. Next, let $\Sigma_{2}=\left\{p_{2}, \boldsymbol{x}_{2}, \boldsymbol{y}_{2}, \boldsymbol{z}_{2}\right\}$ be a

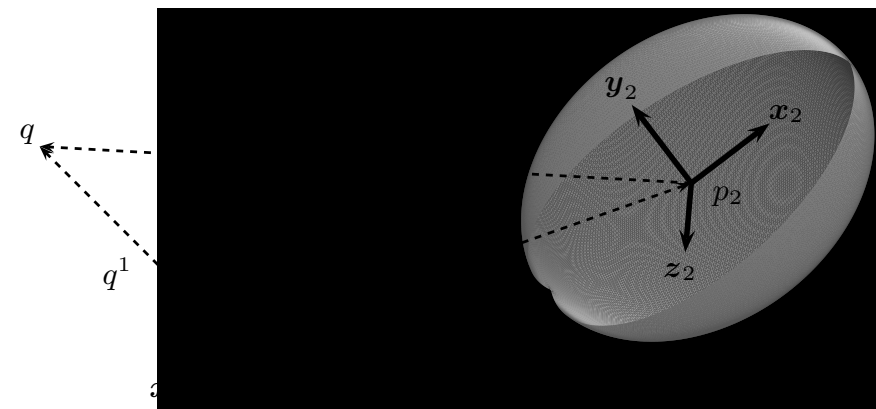

Fig. 1. Two reference frames in $\mathbb{R}^{3}$

reference frame fixed with a moving body. The origin of $\Sigma_{2}$ is the point $p_{2}$, denoted by $p_{2}^{1}$ when expressed with respect to $\Sigma_{1}$. The orientation of $\Sigma_{2}$ is characterized by the 3 -dimensional rotation matrix $\boldsymbol{R}_{2}^{1}$, whose columns are the frame vectors $\left\{\boldsymbol{x}_{2}, \boldsymbol{y}_{2}, \boldsymbol{z}_{2}\right\}$ of $\Sigma_{2}$ expressed with respect to $\Sigma_{1}$. We recall here the definition of the set of rotation matrices in $d$-dimensions, for $d \in\{2,3\}$ :

$$
S O(d)=\left\{\boldsymbol{R} \in \mathbb{R}^{d \times d} \mid \boldsymbol{R} \boldsymbol{R}^{T}=I_{d}, \operatorname{det}(\boldsymbol{R})=+1\right\} .
$$

With these notations, reference frame transformations are described by

$$
q^{1}=\boldsymbol{R}_{2}^{1} q^{2}+p_{2}^{1}, \quad \text { and } \quad \boldsymbol{v}^{1}=\boldsymbol{R}_{2}^{1} \boldsymbol{v}^{2} .
$$

Recall also $\boldsymbol{R}_{2}^{1}=\left(\boldsymbol{R}_{1}^{2}\right)^{T}$. Analogously, we let $S^{i}$ denote the point set $S$ as expressed in the reference frame $\Sigma_{i}$. Finally, if three reference frames $\Sigma_{i}, i \in\{1,2,3\}$, are considered, then simple bookkeeping arguments lead to

$$
\boldsymbol{R}_{2}^{1} \boldsymbol{R}_{3}^{2} \boldsymbol{R}_{1}^{3}=I_{3}, \quad \text { and } \quad \boldsymbol{R}_{2}^{1}=\boldsymbol{R}_{3}^{1} \boldsymbol{R}_{2}^{3} .
$$

Next, it is convenient to present a planar case version of these notions. In the planar case, $p_{1}$ and $p_{2}$ take values in $\mathbb{R}^{2}$, the reference frames consist of only two orthonormal vectors, and the rotation matrices take values in $S O(2)$. It is convenient to identify $\mathbb{R}^{2}$ with the set of complex numbers $\mathbb{C}$ and to denote the unit imaginary number by $\sqrt{-1} \in \mathbb{C}$. If we describe the planar rotation matrix $\boldsymbol{R}_{2}^{1} \in S O(2)$ by its unit-length complex number representation $\exp \left(\theta_{2}^{1} \sqrt{-1}\right)$, with angle $\theta_{2}^{1} \in[-\pi, \pi[$, then the second part of equation (2) reads

$$
\boldsymbol{v}^{1}=\exp \left(\theta_{2}^{1} \sqrt{-1}\right) \boldsymbol{v}^{2}
$$

\section{B. Elements of graph theory}

We review a few useful notions from graph theory [6], [7]. We let $G=(V, E)$ represents an undirected graph $G$, with vertex set $V \triangleq\left\{v_{i}\right\}_{i=1}^{n}$ and edge set $E$ with cardinality m. $G_{d}=\left(V, E_{d}\right)$ defines a directed graph associated to $G$, where $E_{d}$ is an orientation of $E$. We denote a directed edge from vertex $v_{i}$ to $v_{j}$ by $e_{i j}=(i, j)$. If the graph is undirected, $(i, j)$ is equivalent to $(j, i)$.

Definition 1 (Path and cycle) Let $G$ be either a directed or undirected non-empty graph. A path is a non-empty graph
$P=\left(V_{P}, E_{P}\right) \subseteq G$ of the form $V_{P} \triangleq\left\{v_{i}\right\}_{i=1}^{k}$ and $E_{p} \triangleq\left\{\left(j_{i}, j_{i+1}\right)\right\}_{i=1}^{k-1}$, where $\left\{j_{1}, \cdots, j_{k}\right\}$ is a permutation of $\{1, \cdots, k\}$. The vertices $v_{j_{2}}, \cdots, v_{j_{k-1}}$ are the inner vertices of $P$. Furthermore, every sequence of edges that form a closed path in $G$ and do not visit the same node twice, except the start/end node, is called cycle and it is denoted by $\ell$.

The direction of a cycle is the order in which the nodes are visited. We let $\mathcal{L}(G)$ denote the set of all cycles of $G$. We let $|\ell|$ denote the number of edges in the cycle $\ell$. It should be noted that, in a digraph $G_{d}$, the cycle directions are independent of the direction of the individual edges composing the cycles.

Definition 2 (Cycle vector) For $\ell \in \mathcal{L}\left(G_{d}\right)$, the cycle vector is the vector $\mathbf{1}_{\ell} \in\{-1,0,+1\}^{m} \subset \mathbb{R}^{m}$ whose ith entry is +1 if the ith edge belongs to $\ell$ and its orientation is consistent with the orientation of $\ell,-1$ if the ith edge belongs to $\ell$ and its orientation is opposite the orientation of $\ell$, and is 0 otherwise.

Definition 3 (Set of cycle and fundamental cycle vectors) The set of cycle vectors is $L=\left\{\mathbf{1}_{\ell} \mid\right.$ for all $\left.\ell \in \mathcal{L}\left(G_{d}\right)\right\}$. A set of fundamental cycle vectors $L_{f} \subseteq L$ is a subset of $L$ that constitute a base for $L$. The elements of $L_{f}$ are called fundamental cycle vectors.

Given a set of fundamental cycle vectors $L_{f}$, we let $\mathcal{L}_{f}\left(G_{d}\right)$ denote the associated fundamental cycles

$$
\mathcal{L}_{f}(G)=\left\{\ell \in \mathcal{L}\left(G_{d}\right) \mid \mathbf{1}_{\ell} \in L_{f}\right\} .
$$

Definition 4 (Cycle and fundamental cycle matrix) The cycle matrix $C$ of a directed graph $G_{d}$ is the $k \times m$ matrix $C=\left[\mathbf{1}_{\ell_{1}}, \ldots, \mathbf{1}_{\ell_{k}}\right]^{T}$ where $k$ is the dimension of $L$, and $m$ is the number of edges of $G_{d}$. The $r \times m$ matrix $C_{f} \subseteq C$, with $r=\operatorname{dim}\left(L_{f}\right)$, such that each row represents a fundamental cycle vector in $L_{f}$, is called the fundamental cycle matrix:

$$
C_{f}=\left[\mathbf{1}_{\ell_{1}}, \ldots, \mathbf{1}_{\ell_{r}}\right]^{T}, \quad \text { for all } \mathbf{1}_{\ell_{i}} \in L_{f} .
$$

Note that $C_{f}$ is not unique since it depends on the choice of the fundamental cycles vectors, and it is a full rank matrix.

The following result is known from [6].

Theorem 5 (Number of independent cycles) If $G_{d}$ has $n$ vertices and $m$ edges, than the dimension of the fundamental cycle space $L_{f}$ is $m-n+1$, i.e., there are $m-n+1$ independent cycles.

Definition 6 (Plane graph) A graph is called plane graph if it has the following properties:

(i) $V \subseteq \mathbb{R}^{2}$;

(ii) every edge is an arc between two vertices;

(iii) the interior of an edge contains no vertex and no point of any other edge.

The following result is known from [6]. 
Theorem 7 (Number of edges in a plane graph) A plane graph with $n \geq 3$ vertices has at most $3 n-6$ edges.

Definition 8 (Planar network) A planar network is a graph with nodes in $\mathbb{R}^{2}$.

Note not to confuse planar network with planar graph. In literature, the term planar graph defines all the graphs that can be drawn in the plane without edges crossing. This definition is clearly different from our definition of planar network.

\section{NETWORK MODEL AND LOCALIZATION PROBLEMS}

In what follows we describe our notion of network equipped with relative sensors. We consider a group of $n$ nodes in $\mathbb{R}^{d}$, for $d \in\{2,3\}$, and we assume that a reference frame $\Sigma_{i}$ with origin $p_{i}$, for $i \in\{1, \ldots, n\}$, is attached to each node. We assume $p_{i} \neq p_{j}$ for all $i \neq j$. We label the 1st node the reference node.

There might or might not exist a fixed spatial reference frame. If a fixed spatial reference frame exists, then we denote it by $\Sigma_{0}$ with origin $p_{0}$.

\section{A. Relative sensing models}

Each node $i$ activates a sensor that detects the presence and returns a measurement about the relative position of any node inside a given sensor footprint. We let $S_{i} \subset \mathbb{R}^{d}$ be the sensor footprint of node $i$ and $S_{i}^{i}$ be its expression in the $\Sigma_{i}$ frame; we shall assume that all node sensors are equal, so that we write $S_{i}^{i}=S^{i}$. We assume that there exists a map sns: $\mathbb{R}^{d} \rightarrow \mathbb{R}^{k}$, for some $k$, called the sensing function, such that node $i$ acquires the symbol $\operatorname{sns}\left(p_{j}^{i}\right)$ for each node $j \in\{1, \ldots, n\} \backslash\{i\}$ that satisfies $p_{j}^{i} \in S^{i}$. The sensor we will use throughout the paper is the following:

Angle-of-arrival sensing: Node $i$ measures $\operatorname{vers}\left(p_{j}^{i}\right)$, i.e., $\operatorname{sns}\left(p_{j}^{i}\right)=\operatorname{vers}\left(p_{j}^{i}\right) \in \mathbb{R}^{d}$, for all nodes $j$ within a fixed sensing range $r$ from $i$, that is, the footprint $S^{i}$ is a disk of radius $r$ and the function sns returns the spherical coordinates of its argument.

Given the nodes $p_{1}, \ldots, p_{n}$, the directed sensing graph, $G_{d}=\left(V_{S}, E_{d}\right)$ is the directed graph where vertex $v_{i}$ corresponds to node $i$ and the directed edge $(i, j) \in E_{d}$ if $p_{j}^{i} \in S^{i}$, that is, if node $j$ is inside the sensor footprint of node $i$. In what follows, we assume that the sensor footprint $S^{i}$ is a unit-radius disk with $p_{i}$ as the center, so that the sensing graph is the so-called unit-disk geometric graph illustrated in Figure 2. With this assumption, if node $i$ senses node $j$, then node $j$ senses node $i$ as well. Therefore, if $(i, j) \in E_{d}$, then $(j, i) \in E_{d}$ as well. To simplify notation we use an undirected graph $G_{S}=\left(V_{S}, E_{S}\right)$ with vertex set $V_{S}$ and undirected edge set $E_{S}$ satisfying $(i, j) \in E_{S} \Longleftrightarrow(i, j) \in$ $E_{d} \Longleftrightarrow(j, i) \in E_{d}$. We call $G_{S}$ the undirected sensing graph or simply the sensing graph. We further assume that a pair of nodes $i$ and $j$ communicate with each other if and only if they can sense each other, i.e., $(i, j) \in E_{S}$. In summary, the physical components of a relative sensing network consist of $n$ nodes with identifiers in $\{1, \ldots, n\}$, with configurations in $\mathbb{R}^{d} \times S O(d)$, and with relative sensors described by the sensor footprint $S^{i}$ and sensing function sns.

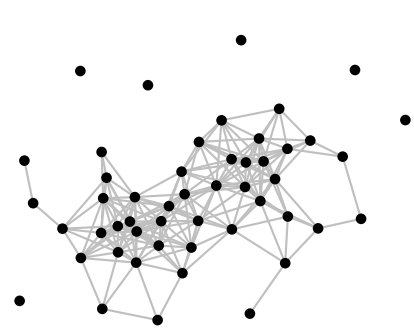

Fig. 2. The disk graph in $\mathbb{R}^{2}$

\section{B. The frame localization problem}

Loosely speaking, we call frame localization the problem of computing the location and orientation of each node of a relative sensing network. Additionally, we call orientation localization the problem of computing the orientation of each node of a relative sensing network. We begin with questions about the uniqueness of these localization problems.

Problem 9 (Frame and orientation localizability) Given a relative sensing network with reference node 1 , provide graph theoretical conditions under which:

(frame localizability:) the reference frames transformations $\left\{\boldsymbol{R}_{i}^{1}, p_{i}^{1}\right\}$, for all $i \in\{2, \ldots, n\}$, are uniquely determined by the relative measurements;

(orientation localizability:) the orientations $\boldsymbol{R}_{i}^{1}$, for all $i \in$ $\{2, \ldots, n\}$, are uniquely determined by the relative measurements.

Second, we are interested in algorithmic matters.

\section{Problem 10 (Centralized and distributed localization)}

Given a frame (respectively, orientation) localizable network, give a centralized or distributed algorithm to compute the reference frames transformation $\left\{\boldsymbol{R}_{i}^{1}, p_{i}^{1}\right\}$ (respectively, the orientations $\boldsymbol{R}_{i}^{1}$ ), for all $i \in\{2, \ldots, n\}$. Give algorithms for both noise-less and noisy sensor measurements.

Finally, for the above questions, we are interested in complexity in arbitrary networks and expected complexity in random geometric networks.

Remark 11 (Data referencing motivation) It is worth remarking that the frame localization problem needs to be solved in relative sensing networks if measurement taken by arbitrary sensors in their respective reference frames need to be expressed (and possibly fused) in a common unique reference frame. Measurements might include positions of targets, environment boundaries, etc.

\section{Preliminary relationships}

In three dimensions, for any sensing and communication undirected edge $(i, j)$, the basic relationship between the relative positions $p_{i}^{j}$ and $p_{j}^{i}$ and the change of frame rotation matrix $\boldsymbol{R}_{j}^{i}$ can be computed from (2) to be

$$
p_{j}^{i}=-\boldsymbol{R}_{j}^{i} p_{i}^{j} .
$$


It is possible to write a normalized version of this equation that applies to angle-of-arrival measurements:

$$
\operatorname{vers}\left(p_{j}^{i}\right)=-\boldsymbol{R}_{j}^{i} \operatorname{vers}\left(p_{i}^{j}\right) .
$$

It is also possible to write a planar version of equation (5), where relative positions are complex numbers and rotations matrices are unit-length complex numbers:

$$
\theta_{j}^{i}=\operatorname{proj}\left(\angle p_{j}^{i}-\angle p_{i}^{j}+\pi\right) .
$$

Remark 12 (Measurements and variables) Recall that the two nodes $i$ and $j$ measure each other's relative positions $p_{j}^{i}$ and $p_{i}^{j}$ (through the sensing function), respectively. The unknown variable in equation (5) is the rotation matrix $\boldsymbol{R}_{j}^{i}$ with $d$ degrees of freedom.

It is possible to parametrize the solutions to equation (6).

Lemma 13 (Feasible orientations) Given unit-length measurements $p_{j}^{i}$ and $p_{i}^{j}$, compute $\boldsymbol{H}_{j}^{i} \in S O(3)$ by

$$
\boldsymbol{H}_{j}^{i}=\exp \left(\alpha_{j}^{i} \widehat{\boldsymbol{e}_{j}^{i}}\right)
$$

where $\boldsymbol{e}_{j}^{i} \in \mathbb{R}^{3}, \alpha_{j}^{i} \in[0, \pi]$ are defined by

$$
\begin{gathered}
\boldsymbol{e}_{j}^{i}= \begin{cases}\operatorname{vers}\left(p_{j}^{i} \times p_{i}^{j}\right), & \text { if } p_{j}^{i} \times p_{i}^{j} \neq \mathbf{0}, \\
\text { any unit-length vector } \perp p_{j}^{i}, & \text { otherwise }\end{cases} \\
\alpha_{j}^{i}=\arctan _{2}\left(\left\|p_{j}^{i} \times p_{i}^{j}\right\|,-p_{j}^{i} \cdot p_{i}^{j}\right) .
\end{gathered}
$$

Then every solution to equation (6) may be written as

$$
\boldsymbol{R}_{j}^{i}=\exp \left(\beta \widehat{\operatorname{vers}\left(p_{j}^{i}\right)}\right) \boldsymbol{H}_{j}^{i},
$$

for an appropriate angle $\beta \in[-\pi, \pi[$.

\section{Planar FRAME LOCALIZATION}

A. Orientation localizability with angle-of-arrival sensors

Our first localizability result follows.

Theorem 14 (Orientation localizability for planar networks with angle-of-arrival sensing) Consider a relative sensing network with $d=2$ (i.e., a planar network) and with noiseless angle-of-arrival sensing. The following statements are equivalent:

(i) the sensing graph is connected, and

(ii) the network is orientation localizable.

Proposition 15 A network with only range measurements is not orientation localizable.

Proof: The range measurement is independent of the reference frame. That is, given a fixed geometry of a network, each node may have an infinite number of orientations.

\section{Proposition 16 (Sufficient conditions for localizability)}

A network with $n$ nodes capable of angle-of-arrival measurement is both frame localizable and orientation localizable if the sensing graph is rigid and at least one of the edge lengths is known.
B. Orientation localization with noisy angle-of-arrival sensors

Now we follow Theorem 14, and we consider a planar network with angle-of-arrival sensing. We assume that, for each undirected edge $(i, j)$ of the sensing graph, nodes $i$ and $j$ measure, respectively, the angles

$$
\angle p_{j}^{i}+n_{j}^{i} \quad \text { and } \quad \angle p_{i}^{j}+n_{i}^{j},
$$

where we suppose the noises $n_{i}^{j}$ and $n_{j}^{i}$ to be independent, Gaussian random variables with zero mean and variance $\sigma$. Therefore, for each undirected edge $(i, j)$, we can measure only

$$
y_{j}^{i}=\operatorname{proj}\left(\left(\angle p_{j}^{i}+n_{j}^{i}\right)-\left(\angle p_{i}^{j}+n_{i}^{j}\right)+\pi\right),
$$

and not the true relative orientation $\theta_{j}^{i}$ as in equation (7).

Remark 17 (Redundant measurements in cycles) If the sensing graph is a tree, then there is no redundant measurement and we cannot reduce the effect of measurement noise on our angle estimates. However, for every cycle in the network, we can enforce a cycle constraint (see equation (3)). We formalize this statement as follows.

Let $G_{S}=\left(V_{S}, E_{S}\right)$ be the undirected sensing graph with $n$ nodes and $m$ edges. We assign a direction to each edge in $E_{S}$ in the following way: the direction is from $i$ to $j$ if $i<j$. Noting that this direction assignment is different from/independent of the sensing/communication relations, let us denote the directed graph obtained, by $G_{d}=\left(V_{S}, E_{d}\right)$. Consider the oriented edge $e=(j, i) \in E_{d}$, with $i>j$. Let $\psi_{e}$ denote the estimate of the true relative angle associated to $e, \theta_{e}=\theta_{i}^{j}$. Let $\psi \in \mathbb{R}^{m}$ denote the vector of angle estimates for all the edges of the graph. Analogously, we let $y$ denote the measurement vector with components $y_{e}=y_{i}^{j}$, for $i>j$. For $\ell \in \mathcal{L}\left(G_{d}\right)$, the cycle error $\epsilon_{\ell}$ at $\psi$ is

$$
\boldsymbol{\epsilon}_{\ell}=\operatorname{proj}\left(\mathbf{1}_{\ell} \cdot \psi\right)
$$

where the map proj: $\mathbb{R} \rightarrow[-\pi, \pi[$ is defined in (1) and the map proj: $\mathbb{R}^{n} \rightarrow\left[-\pi, \pi\left[^{n}\right.\right.$ is defined by

$$
\operatorname{proj}\left(\left[x_{1}, \ldots, x_{n}\right]^{T}\right)=\left[\operatorname{proj}\left(x_{1}\right), \ldots, \operatorname{proj}\left(x_{n}\right)\right]^{T} .
$$

Note that

$$
\operatorname{proj}\left(\mathbf{1}_{\ell} \cdot \psi\right)=\operatorname{proj}\left(\sum_{f \in \ell} \pm \psi_{f}\right),
$$

where \pm indicates whether or not the direction of the edge $f$ is concordant with the direction of the cycle $\ell$ which $f$ belongs to.

In what follows, we aim to solve the following least-square estimation problem:

$$
\min _{\psi}\|\psi-y\|^{2}
$$

subj. to $\operatorname{proj}\left(\mathbf{1}_{\ell} \cdot \psi\right)=0$, for all $\ell \in \mathcal{L}\left(G_{d}\right)$.

Note that the optimal $\psi$ lives in a set of countable affine subspaces; once the optimal affine subspace is determined, the optimal estimate is computed via a linear projection. 


\section{Optimal estimation in a ring}

Now, suppose the sensing graph $G_{S}$ is a ring with nodes $\{1, \ldots, n\}$ and with undirected edges $(i,(i+1) \bmod n)$, for $i \in\{1, \ldots, n\}$. In what follows, we write $(i+1)$ to denote $(i+1) \bmod n$. Compute a set of angle estimates $\psi_{i+1}^{i}$ by

$$
\psi_{i+1}^{i}=y_{i+1}^{i}-\frac{1}{n}\left(\operatorname{proj}\left(\sum_{i=1}^{n-1} y_{i+1}^{i}-y_{n}^{1}\right)\right),
$$

and $\psi_{n}^{1}=-\psi_{1}^{n}$. Equation (12) can be written in vectorial form as

$$
\psi=y-\mathbf{1}_{\ell} \frac{1}{n} \operatorname{proj}\left(\mathbf{1}_{\ell} \cdot y\right),
$$

where $y=\left[y_{2}^{1}, y_{3}^{2}, \cdots, y_{n}^{1}\right]^{T}$ and $\psi=\left[\psi_{2}^{1}, \psi_{3}^{2}, \cdots, \psi_{n}^{1}\right]^{T}$. This affine map is a projection onto one of the affine subspaces that describe the constraint in the optimization problem (11).

Theorem 18 (Solution to the least squares) The angle estimates computed in equation (12) are the solution to the least squares estimation problem (11).

It is interesting to note that equation (13) is a particular case of the Kaczmarz's projection method [8] for solving a system of linear equations through iterative projections.

\section{An iterative estimation algorithm for arbitrary graphs}

We now consider an arbitrary network $G_{d}$ with set of cycles $\mathcal{L}\left(G_{d}\right)$ and we propose a natural generalization of the optimal estimation algorithm (13). Let $\hat{\mathcal{L}} \subseteq \mathcal{L}\left(G_{d}\right)$ be a subset of the cycle set, and let $\psi_{e}$ denote the estimate of the angle associated to the edge $e$. For $0<\kappa \ll 1$, consider the following "cycle-distributed" discrete-time system:

$$
\begin{aligned}
\psi_{e}(0) & =y_{e}, \\
\psi_{e}(t+1) & =\psi_{e}(t)-\kappa \sum_{\ell \in \hat{\mathcal{L}}: e \in \ell}\left(\mathbf{1}_{\ell} \cdot \boldsymbol{e}_{e}\right) \operatorname{proj}\left(\mathbf{1}_{\ell} \cdot \psi(t)\right),
\end{aligned}
$$

where $\boldsymbol{e}_{i}$ is the $m$-dimensional vector whose $i$ th entry is 1 , and all the other entries are equal to zero. We will often focus our attention to the case where the set of cycles $\hat{\mathcal{L}}$ is a set of fundamental cycles $L_{f}$.

Theorem 19 (Exponential convergence) Consider a planar relative sensing network $\mathcal{N}$ with noisy angle-of-arrival sensing and sensing graph $G_{S}=\left(V_{S}, E_{S}\right)$ with $n$ vertices and $m$ edges, and its associated directed graph $G_{d}=$ $\left(V_{S}, E_{d}\right)$. Let $L_{f}$ be a fundamental cycle set for the digraph with associated fundamental cycle matrix $C_{f}$. The solution of the discrete-time system (14) with $\hat{\mathcal{L}}=\mathcal{L}_{f}$ converges exponentially fast with exponential converges factor $\rho=$ $(1-\kappa)^{2}$, to the set of angles with zero cycle error for $\kappa<2 /\left(1+\lambda_{\max }(F)\right)$, where $F=C_{f} C_{f}^{T}$, and $\lambda_{\max }(F)$ is the maximum eigenvalue of $F$.

At this time, it is not known whether the proposed algorithm computes the optimal least-square estimate of the unknown angles. Numerical experiments in Section $\mathrm{V}$ illustrate however its compelling performance in this regard.

\section{E. Some remarks on complexity}

in order to speed up the exponential convergence factor $\rho$ of algorithm (14), it is desirable to maximize $\kappa$. To compute the largest possible $\kappa$ that guarantees convergence, it is natural to ask how to choose $C_{f}$, i.e., how to choose the fundamental cycle set in order to minimize the maximum eigenvalue of the matrix $F=C_{f} C_{f}^{T}$. At this time, we only provide the following conservative analysis. One can see that $\operatorname{trace}(F)=\sum_{i \in\{1, \ldots, r\}}\left|\ell_{i}\right|$, and, since $\lambda_{\max }(F)<$ trace $(F)$, exponential convergence of algorithm (14) is guaranteed if

$$
\kappa<\frac{2}{1+\sum_{i \in\{1, \ldots, r\}}\left|\ell_{i}\right|} .
$$

From Theorem 5, we know the fundamental cycle space has rank $m-n+1$ in a digraph with $n$ nodes and $m$ edges. In the worst-case, it is possible for a digraph to have order $n^{2}$ edges and it is certainly true that each cycle has at most order $n$ edges. Therefore, in the worst-case, we can only choose

$$
\kappa \in O\left(\frac{1}{n^{3}}\right) .
$$

Suppose now instead that (i) the graph is planar, so that it has at most $3 n-6$ edges by Theorem 7 , and that (ii) we consider only cycles with bounded length (e.g., in a planar graph that is a triangulation, one can choose a fundamental cycle set with all cycles of length 3 ). Then we can choose

$$
\kappa \in O\left(\frac{1}{n}\right) .
$$

More generally, how to choose a fundamental cycle set to minimize the sum of cycle lengths is an optimization problem known as the minimum cycle basis problem. In the beautiful work of Elkin and coworkers [9], the authors construct a fundamental cycle basis for an unweighted undirected graph of length $O\left(n^{2}\right)$.

\section{Simulations}

We provide some simulations to illustrate the performance of the proposed distributed algorithm considering $\hat{\mathcal{L}}$ as a set of independence cycles. We consider arbitrary network configurations with fixed node positions and varying sensing footprints, e.g., see Figure 3. Different number of edges in the network lead to different number of independent loops. As illustrated by the plot in Figure 4, the mean-square error $\|\psi-\theta\|^{2}$ is smaller than $\|y-\theta\|^{2}$ and decreases as the number of independent loops increases. Convergence of (14) is shown in Figure 5.

\section{CONCLUSIONS AND FUTURE WORK}

This paper introduces the frame localization problem in a connected network. For the orientation localization problem with angle-of-arrival (bearing) sensors, we developed an algorithm that reduces the effect of noise. Our algorithm computes the correct least-square estimate for ring networks in one step. Our algorithm is proved to converge exponentially fast and is validated it through some simulations. We are currently extending the work in several directions. First, we want to improve the efficiency of the orientation localization algorithm and either show its least-square 


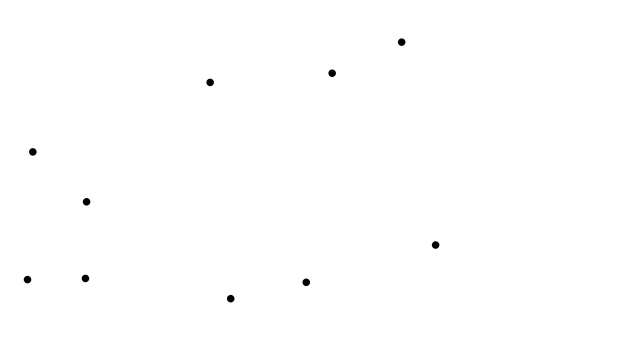

(a)

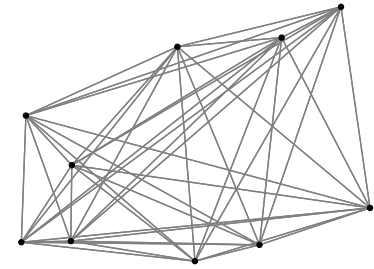

(b)

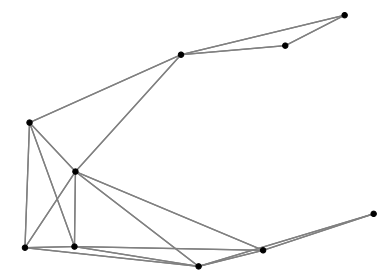

(c)
Fig. 3. Plot (a) refers to a configuration of $n=10$ points. By considering different sensing footprints, in plot (b) it is shown the complete graph with 36 independent loops, and in (c) we show the same nodes configuration but with 10 independent loops.

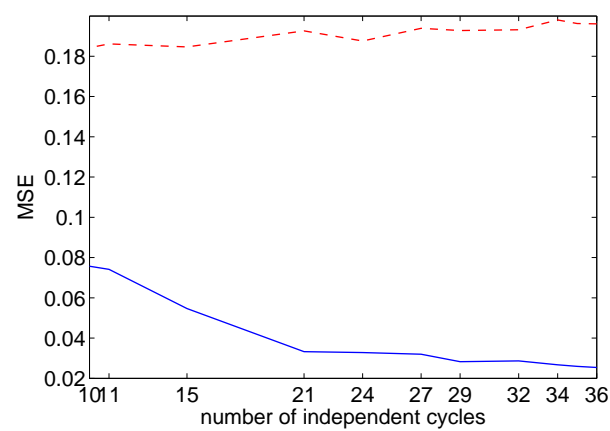

Fig. 4. Evolution of the average MSE of $\psi$ and $y$ for network in Figure 3 and measurement noise variance $\sigma^{2}=0.1$ as the number of edges in the graph changes. The dashed and continuous lines represent the evolution respectively of $\|y-\theta\|^{2}$ and $\|\psi-\theta\|^{2}$.

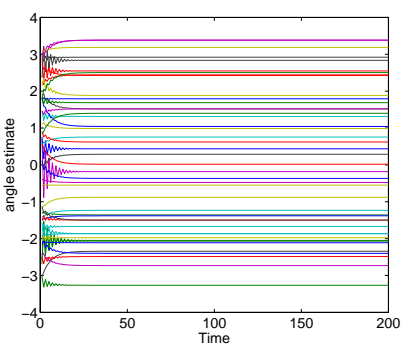

(a)

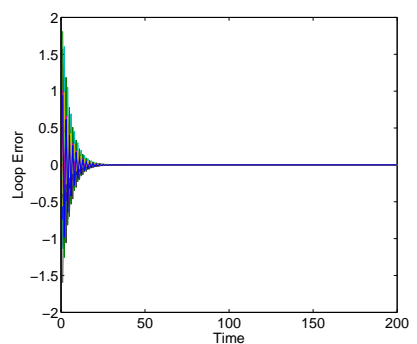

(b)
Fig. 5. This plot refers to the complete graph in Figure 3 (b), using (14) and noise variance $\sigma^{2}=0.01$. In the first plot from left it is shown the angle estimate for every edge. In the second plot it is shown the loop error for every loop.

optimality or modify it to achieve least-square optimality. Second, we aim to study orientation localization in mixed setups with angle-of-arrival as well as exploring the case with the use of distance measurements. Third, we plan to

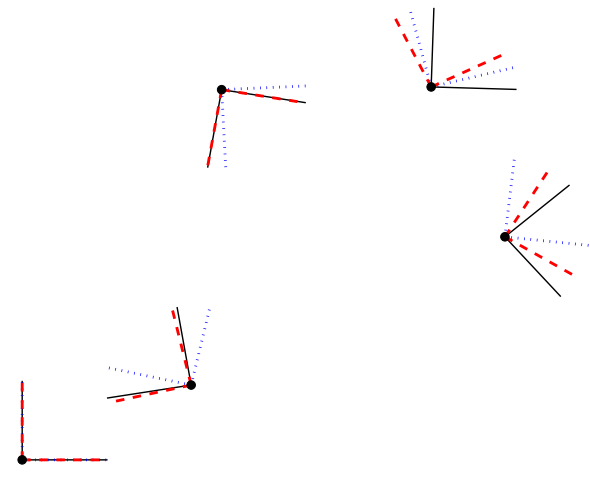

Fig. 6. This figure shows a complete network performing selflocalization considering a set of independent cycles. The solid lines are the true frames. The dotted lines are the measured frames (with respect to the pre-specified frame in the bottom left corner). The dashed line are the estimated frame computed by algorithm (14).

address the problem of position localization, defined earlier in this paper. Fourth and final, we aim to formulate conditions for frame localization in three dimensions. In particular, we are investigating orientation localizability for three nodes in 3D space, to subsequently expand the work towards localizability for arbitrary networks.

\section{ACKNOWLEDGMENTS}

This material is based upon work supported in part by NSF Award CMS-0626457 and ONR Award N00014-071-0721. National ICT Australia - NICTA is funded by the Australian Government as represented by the Department of Broadband, Communications and the Digital Economy and the Australian Research Council through the ICT Centre of Excellence program.

\section{REFERENCES}

[1] N. Bulusu, J. Heidemann, and D. Estrin, “Adaptive beacon placement,' in International Conference on Distributed Computing Systems, Mesa, AZ, Apr. 2001, pp. 489-498.

[2] X. S. Zhou and S. I. Roumeliotis, "Determining the robot-to-robot relative pose using range-only measurements," in IEEE Int. Conf. on Robotics and Automation, Roma, Italy, Apr. 2007, pp. 4025-4031.

[3] N. Trawny, X. S. Zhou, K. X. Zhou, and S. I. Roumeliotis, "3D relative pose estimation from distance-only measurements," in IEEE/RSJ Int. Conf. on Intelligent Robots \& Systems, San Diego, CA, Oct. 2007, pp. 1071-1078.

[4] J. Aspnes, T. Eren, D. K. Goldenberg, A. S. Morse, W. Whiteley, Y. R. Yang, B. D. O. Anderson, and P. Belhumeur, "A theory of network localization," IEEE Transactions on Mobile Computing, vol. 5, no. 12, pp. 1663-1678, 2006.

[5] G. Piovan, I. Shames, B. Fidan, F. Bullo, and B. D. O. Anderson, "On frame and orientation localization for relative sensing networks," Center for Control, Dynamical Systems and Computation. University of California at Santa Barbara, Tech. Rep. CCDC-08-0905, 2008, available electronically at http://ccdc.mee.ucsb.edu.

[6] R. Diestel, Graph Theory, 2nd ed., ser. Graduate Texts in Mathematics. New York: Springer Verlag, 2005, vol. 173.

[7] L. R. Foulds, Graph Theory Applications, ser. Universitext. New York: Springer Verlag, 1995.

[8] S. Kaczmarz, "Approximate solution of systems of linear equations (english translation)," International Journal of Control, vol. 57, no. 6 , pp. 1269-1271, 1993.

[9] M. Elkin, C. Liebchen, and R. Rizzi, "New length bounds for cycle bases," Information Processing Letters, vol. 104, no. 5, pp. 186-193, 2007. 